\title{
Expression of ADAM12 is regulated by E2F1 in small cell lung cancer
}

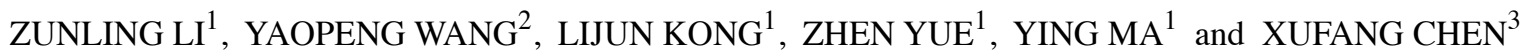 \\ ${ }^{1}$ Department of Biochemistry and Molecular Biology, Binzhou Medical University, Yantai, Shandong 264003; \\ ${ }^{2}$ Department of Thoracic Surgery, Affiliated Hospital of Qingdao University, Qingdao, Shandong 266071; \\ ${ }^{3}$ Department of Oncology, Yantai Affiliated Hospital of Binzhou Medical University, Yantai, Shandong 264199, P.R. China
}

Received August 28, 2015; Accepted September 4, 2015

DOI: $10.3892 /$ or.2015.4317

\begin{abstract}
Our previous study reported that $A D A M 12$ was highly expressed in small cell lung cancer (SCLC) and could be an effective marker for diagnosis and prognosis. Yet, the reason for the high expression of ADAM12 in SCLC requires further elucidation. Transcription factor $E 2 F 1$ has been receiving increasing attention due to the complexity and diversity of its function in cancer. In the present study, the expression of $A D A M 12$ was significantly decreased following silencing of $E 2 F 1$ expression by siRNA, thus indicating that $E 2 F 1$ may regulate the expression of $A D A M 12$ at the level of transcription. Chromatin immunoprecipitation-to-sequence analysis identified three binding sites for $E 2 F 1$ in the locus for ADAM12. They were Chr10: 128010444-128011026, located in the intron of $A D A M 12$, named seq0; Chr10: $128076927-128078127$, located in the promoter of $A D A M 12$, named seq1; and Chr10: 128086195-128086876, located in the upstream $20 \mathrm{~kb}$ from the transcription start site of $A D A M 12$, named: seq2. Dual-luciferase reporter experiments revealed that seq 1 not seq 0 and seq 2 was able to promote the expression of luciferase. Notably, co-transfection of E2F1 significantly increased the activity of seq1 not seq0 and seq2, but quantitative polymerase chain reaction results showed that seq0, seq1 and seq 2 could recruit $E 2 F 1$, indicating that the influence of $E 2 F 1$ in regulating the expression of $A D A M 12$ was complex. Sequence analysis clarified that seq1 was a part of the ADAM12 promoter, yet the functions of seq 0 and seq2 were unknown. Fusion fragments containing seq0-seq1 or seq2-seq1 were analyzed in luciferase constructs. Compared with seq1 alone, the activities of these fusion fragments were non-significantly reduced. The activities of fusion fragments
\end{abstract}

Correspondence to: Professor Xufang Chen, Department of Oncology, Yantai Affiliated Hospital of Binzhou Medical University, 717 Jinbu Street, Muping, Yantai, Shandong 264199, P.R. China E-mail: becky6280@163.com

Abbreviations: ADAM, A disintegrin and metalloproteinase; SCLC, small cell lung cancer

Key words: ADAM12, gene expression, E2F1, small cell lung cancer were significantly decreased following co-transfection with $E 2 F 1$. Thus, the present findings support the conclusion that the $E 2 F 1$ transcription factor regulates the expression of $A D A M 12$ by binding differential cis-acting elements.

\section{Introduction}

$A D A M 12$ is a disintegrin and metalloproteinase family member that plays important roles in embryonic development, acting on multiple processes including cell adhesion and cell movement (1). In the majority of normal adult tissues, the expression of $A D A M 12$ is extremely low (2), but increases in certain pathological conditions, including carcinogenesis (3), osteoarthritis (4) and cardiac hypertrophy (5). ADAM12 has been used as a marker for the diagnosis of breast (6) and prostate cancer (7). Our previous study showed that ADAM12 was highly expressed in small cell lung cancer (SCLC) and can be considered as an effective marker for diagnosis and prognosis (8), yet the reasons for the high level of expression of $A D A M 12$ in SCLC remain unknown.

Studies of the regulation of $A D A M 12$ expression have mainly been focused at the level of transcription. Transforming growth factor- $\beta$ (TGF $\beta$ ) was found to induce the expression of $A D A M 12$ by activating the PI3K and MAPK signaling pathways (9). Z-DNA-binding protein was able to bind a negative element in the 5'-untranslated region of the ADAM12 gene to repress transcription of $A D A M 12$ in numerous tissues (10). The nuclear factor (NF) $\kappa \mathrm{B}$ signaling pathway was able to promote the expression of $A D A M 12$ by inhibiting the expression of $m i R-29(11,12)$. Our previous research showed that $p 65$ was highly expressed and regulated by E2F1 in SCLC (13); therefore we speculated that the transcription factor $E 2 F 1$ may be a significant factor that controls the expression of $A D A M 12$ in SCLC. In the present study, the mechanism by which $E 2 F 1$ regulates the expression of $A D A M 12$ was explored, and $E 2 F 1$ was found to bind to the promoter and other $c i s$-acting elements to regulate the expression of ADAM12 in SCLC.

\section{Materials and methods}

Reagents and antibodies. RPMI-1640 medium was purchased from HyClone, GE Healthcare Life Sciences (Logan, UT, USA). Fetal bovine serum (FBS), siRNA targeting E2F1, 
scrambled siRNA and Lipofectamine 2000 were purchased from Invitrogen Co. (Carlsbad, CA, USA). Penicillin and streptomycin were purchased from Luye Pharmaceutical Co., Ltd. (Yantai, Shandong, China). Protein A/G beads, normal mouse $\operatorname{IgG}$ and a mouse anti-E2F1 monoclonal antibody (sc-251) were purchased from Santa Cruz Biotechnology, Inc. (Dallas, TX, USA). A chromatin immunoprecipitation (ChIP) assay kit (17-295) and ChIP grade mouse anti-E2FI monoclonal antibody (17-10061) were purchased from Merck Millipore (Billerica, MA, USA). The goat anti-ADAM12 polyclonal antibody (AF1025a) was purchased from Abgent Co., Ltd. (Suzhou, China). Goat anti-mouse and rabbit anti-goat secondary antibody with HRP, and the DAB coloring kit were purchased from Beijing Zhongshan Jinqiao Biotechnology Co., Ltd. (Beijing, China). A dual-luciferase analysis kit (E1980) was purchased from Promega Corporation (Madison, WI, USA). FastDigest enzymes including NheI, BglII, KpnI and $M l u \mathrm{I}$ were purchased from Thermo Fisher Scientific Co. (Waltham, MA, USA). A gel extraction kit was purchased from Takara Technology Co. (Dalian, China).

Cell culture and tissue samples. Human SCLC cell lines H1688 and H446, and human lung adenocarcinoma cell line A549 were preserved by our laboratory (Shandong Province Key Laboratory of Tumor Molecular Biology, Binzhou Medical University). All cells were cultured at $37^{\circ} \mathrm{C}$ in humidified 95\% air and 5\% $\mathrm{CO}_{2}$ in RPMI-1640 medium supplemented with $10 \%$ FBS, $100 \mathrm{U} / \mathrm{ml}$ penicillin and $100 \mu \mathrm{g} / \mathrm{ml}$ streptomycin. Forty SCLC tissue samples were obtained from Yantai Affiliated Hospital of Binzhou Medical University from January to December 2013. All samples were biopsy samples, and all patients voluntarily provided informed consent. The present study was approved by the Medical Ethics Committee of Binzhou Medical University (no. 2013007). The patient information is listed in Table I.

ChIP-to-sequence. ChIP was conducted according to the manual provided with the ChIP assay kit (13). In brief, $5 \times 10^{7}$ cells were fixed using $1 \%$ formaldehyde and were subsequently incubated in SDS lysis buffer. Ultrasound was used to fragment the genomic DNA, and the sample was pretreated with protein $\mathrm{A} / \mathrm{G}$ beads, and then centrifugation $\left(2,000 \mathrm{rpm}, 4^{\circ} \mathrm{C}\right)$. The protein beads were then removed. The resulting sample was divided into two parts. One part was incubated overnight with the ChIP grade mouse anti-E2FI monoclonal antibody $(4 \mu \mathrm{g})$, and the other with normal mouse $\operatorname{IgG}(4 \mu \mathrm{g})$. On the following morning, the protein $\mathrm{A} / \mathrm{G}$ beads were added and incubated for $2 \mathrm{~h}$ at $4^{\circ} \mathrm{C}$. The resulting antigenantibody-protein bead complexes were reverse crosslinked in the presence of salt at a high concentration $(5 \mathrm{M}, \mathrm{NaCl})$. DNA fragments were purified and sequenced (BGI Co., www.genomics.cn). The data processing was reported in our previous study (13).

Immunohistochemistry $(I H C)$. IHC was carried out according to the protocols of our laboratory $(8,13)$. In brief, the sections were dewaxed and rehydrated in a series of alcohols to water. Antigens were retrieved by heating the sections in citrate buffer $(0.01 \mathrm{M}, \mathrm{pH}=6.0)$ for $45 \mathrm{~min}$ at $95^{\circ} \mathrm{C}$ in a boiler. The sections were subsequently incubated with the primary antibodies
Table I. Basic information of the SCLC patients.

\begin{tabular}{lrc}
\hline Characteristics & No. & Percent (\%) \\
\hline Age (years) & & \\
$\geq 60$ & 34 & 85.0 \\
$<60$ & 6 & 15.0 \\
Gender & & \\
Male & 29 & 72.5 \\
Female & 11 & 27.5 \\
Smoking history & & \\
Smoker & 33 & 82.5 \\
Non-smoker & 7 & 17.5 \\
Clinical phage & & \\
LD & 5 & 12.5 \\
ED & 35 & 87.5 \\
\hline
\end{tabular}

$\mathrm{LD}$, limited disease; ED, extensive disease.

overnight at $4^{\circ} \mathrm{C}$. The dilution of primary antibodies was 1:50 for $E 2 F 1$ and 1:200 for ADAM12. On the following day, all the sections were incubated with a secondary horseradish peroxidase (HRP)-conjugated antibody, and a brown color reaction was developed using the DAB kit. Sections were counterstained with hematoxylin, differentiated, dehydrated, cleared and mounted in neutral gum. The IHC scores were assessed according to our previous studies $(8,13)$.

Reverse transcription-quantitative polymerase chain reaction (RT-qPCR). RT-qPCR was performed according to our previous studies $(8,13)$. The primers for each target gene are listed in Table II.

Western blotting. Western blotting was performed according to our previous studies $(8,13)$. Cells were lysed, and $50 \mu \mathrm{g}$ of protein was loaded and separated on $10 \%$ polyacrylamide gels (70 V for $30 \mathrm{~min} ; 140 \mathrm{~V}$ for $70 \mathrm{~min} ; 180 \mathrm{~V}$ for $10 \mathrm{~min}$ ). Proteins were subsequently transferred to $\mathrm{NC}$ membranes by wet transfer (300 mA for $150 \mathrm{~min}$ ), which were blocked with 5\% skimmed milk powder, prior to incubation with the primary antibodies overnight at $4^{\circ} \mathrm{C}$. On the following morning, the membranes were washed four times (three times in TBS buffer, one time in TBST buffer), incubated with HRP-conjugated secondary antibodies (goat anti-mouse for $E 2 F 1$ and rabbit anti-goat for ADAM12, 1:5,000), washed four times and exposed to X-ray film. The dilution of primary antibodies was as follows: 1:100 for $E 2 F 1,1: 200$ for $A D A M 12$.

siRNA treatment. siRNAs targeting E2F1 and a scramblecontrol siRNA were used to assess the relevance of $E 2 F 1$ to the expression of ADAM12 (13). Cells $\left(1 \times 10^{5}\right)$ were cultured into a 6-well plate and transfected with Lipofectamine 2000. The procedure was performed according to previously described methods $(8,13)$.

Assembly of luciferase reporter constructs. Genomic DNA was extracted from the H1688 cells, and the fragments 
Table II. Primers of the target genes for RT-qPCR.

\begin{tabular}{|c|c|c|c|}
\hline Name & Primer sequences & Length (bp) & $\operatorname{Tm}\left({ }^{\circ} \mathrm{C}\right)$ \\
\hline \multirow[t]{2}{*}{ Seq0 } & F ATTCAGGAAGACGGGTGGCT & & \\
\hline & $\mathrm{R}$ TGGTAACCCATCCATTAAGCGG & 70 & 60 \\
\hline \multirow[t]{2}{*}{ Seq1 } & F GGTGGTCCTAGGTCTGAGCA & & \\
\hline & $\mathrm{R}$ TCAGTTTCCCACAATGCGTG & 81 & 60 \\
\hline \multirow[t]{2}{*}{ Seq2 } & F GCACTCAGCGTCCTATCTGT & & \\
\hline & R AAAGTACGCTTGCCAGACCA & 72 & 60 \\
\hline \multirow[t]{2}{*}{$\mathrm{E} 2 \mathrm{~F} 1$} & F CATCAGTACCTGGCCGAGAG & 118 & 60 \\
\hline & $\mathrm{R}$ TGGTGGTCAGATTCAGTGAGG & & \\
\hline \multirow[t]{2}{*}{ ADAM12 } & F GCAGTTTCACGGAAACCCAC & & \\
\hline & R ACACGTGCTGAGACTGACTG & 131 & 60 \\
\hline
\end{tabular}

Table III. Primers of the target fragments for luciferase reporter constructs.

\begin{tabular}{|c|c|c|c|}
\hline Fragment & & Primers & $\mathrm{E}$ \\
\hline \multirow[t]{2}{*}{ Seq0 } & $\mathrm{F}$ & ACTGGTACCAGTATGTACAAATGAAGTGTCATG & KpnI \\
\hline & $\mathrm{R}$ & AAT $\underline{A C G C G T A G A C C A T G C G G T T C C C A}$ & MluI \\
\hline \multirow{2}{*}{ Seq1 } & $\mathrm{F}$ & ACT $G C T A G C G T G C T C C G T C A G G A A T C G G T$ & NheI \\
\hline & $\mathrm{R}$ & ACTAGATCTTTCTGGCACAAGCCAGCCTT & $B g l \mathrm{II}$ \\
\hline \multirow[t]{2}{*}{ Mut-Seq1 } & $\mathrm{P} 1$ & TCTTATTAaaaaGGAAC & \\
\hline & $\mathrm{P} 2$ & GTTCC $t t t t$ TAATAAGA & \\
\hline \multirow[t]{2}{*}{ Seq2 } & $\mathrm{F}$ & AAT $\underline{G T A C C G G G C A G T T G G C T C T G T T A}$ & KpnI \\
\hline & $\mathrm{R}$ & AAT $\underline{A C G C G T A A C C C A A A T A G C C C T G C C}$ & Mlu $\mathrm{I}$ \\
\hline
\end{tabular}

Italicized and underlined sections indicate FastDigest enzyme sites.

(Chr10: 128010444-128011026, located in the intron of ADAM12, named seq0; Chr10: 128076927-128078127, located in the promoter of $A D A M 12$, named seq1; Chr10: 128086195-128086876, located in the upstream $20 \mathrm{~kb}$ from transcription start site of $A D A M 12$, named seq2) pulled down by $E 2 F 1$ were amplified by PCR using primers with the sequences shown in Table III. These PCR fragments and the pGL3-basic promoter-less vector were digested with FastDigest $N h e \mathrm{I}, B g l \mathrm{II}$ for seq1, KpnI and $M l u \mathrm{I}$ for seq0 and seq2. The digested fragments were extracted using a gel extraction kit and ligated using T4 DNA ligase to generate three luciferase reporter constructs. The luciferase reporter vector containing a mutant $E 2 F 1$ binding site was constructed by overlap PCR using the primers listed in Table III. These three luciferase reporter vectors were digested with FastDigest $K p n I$ and $M l u I$, and the seq 0 and seq 2 fragments were ligated into the digested seq1 vector using T4 DNA ligase to generate the fusion luciferase reporter constructs containing seq0-seq1 and seq2-seq1. All the constructs were verified by sequencing (BioSune Co., Jinan, China).

Luciferase reporter analysis. H1688, H446 and A549 cells were transfected with $0.5 \mu \mathrm{g}$ luciferase reporter vector, $0.3 \mu \mathrm{g}$ E2F1 expression vector or pcDNA3.1 and 0.02 $\mu \mathrm{g}$ pRL-TK
Renilla reniformis luciferase. Luciferase activity was analyzed with dual-luciferase assay kits according to the instruction manual.

Statistical analysis. All the data were analyzed using SPSS 17.0 software (SPSS, Inc., Chicago, IL, USA) and paired $\mathrm{t}$-tests were used to assess the significance of the differences in expression among the groups. $\mathrm{P}<0.05$ was considered to indicate a statistically significant difference.

\section{Results}

ADAM12 is highly expressed in SCLC samples in which E2F1 is strongly positive. Our previous results found that $A D A M 12$ and E2F1 were highly expressed in SCLC tissue samples, respectively $(8,13)$. NF- $\kappa \mathrm{B}$ induced the expression of $A D A M 12(11,12)$ and p65 was highly expressed and regulated by $E 2 F 1$ in the SCLC samples (13), which indicated that $E 2 F 1$ may be a significant factor for promoting the expression of $A D A M 12$. Since the tissues used for detection in our previous studies were from differential hospitals $(8,13)$, it was unconvincing that E2F1 may regulate the expression of $A D A M 12$. In order to solve this issue, an additional 40 SCLC tissue samples were obtained. IHC results revealed that negative/ 
Table IV. Expression levels of E2F1 and ADAM12 in 40 SCLC tissue samples.

\begin{tabular}{|c|c|c|c|c|c|c|c|}
\hline \multicolumn{4}{|c|}{$\mathrm{E} 2 \mathrm{~F} 1$} & \multicolumn{4}{|c|}{ ADAM12 } \\
\hline \multicolumn{2}{|c|}{ Negative } & \multicolumn{2}{|c|}{ Positive } & \multicolumn{2}{|c|}{ Negative } & \multicolumn{2}{|c|}{ Positive } \\
\hline Negative & Weak & Moderate & Strong & Negative & Weak & Moderate & Strong \\
\hline 2 & 3 & 12 & 22 & 5 & 4 & 11 & 19 \\
\hline
\end{tabular}

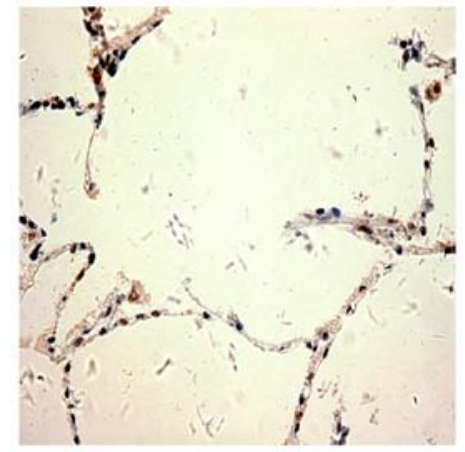

Normal tissue staining

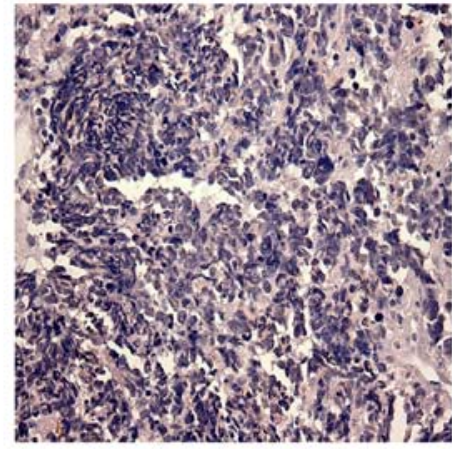

Negative control

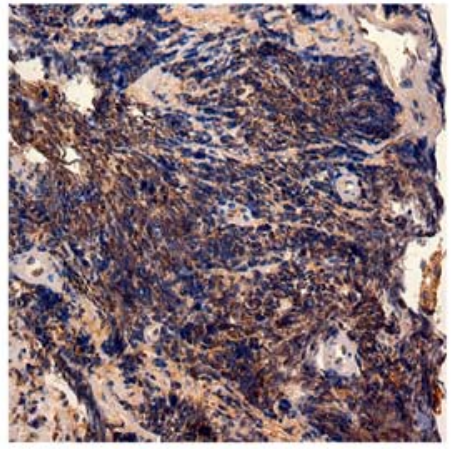

VEGFR

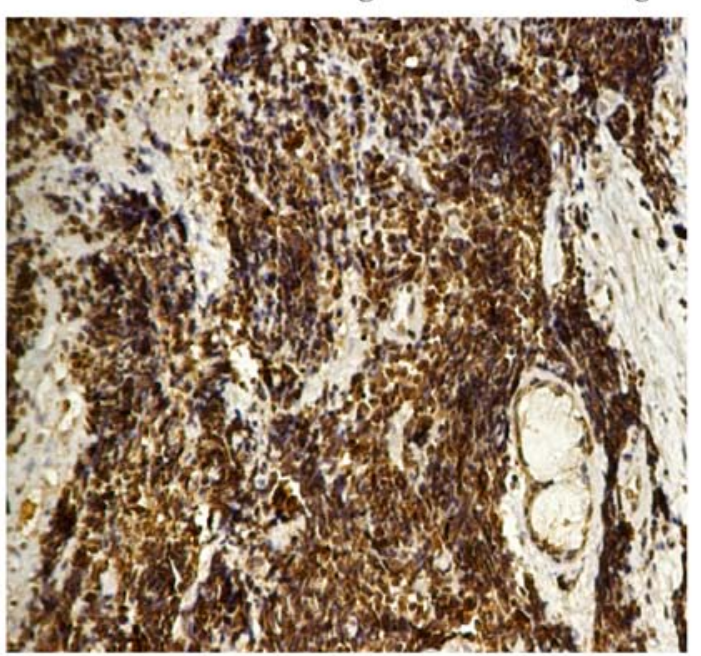

ADAM12

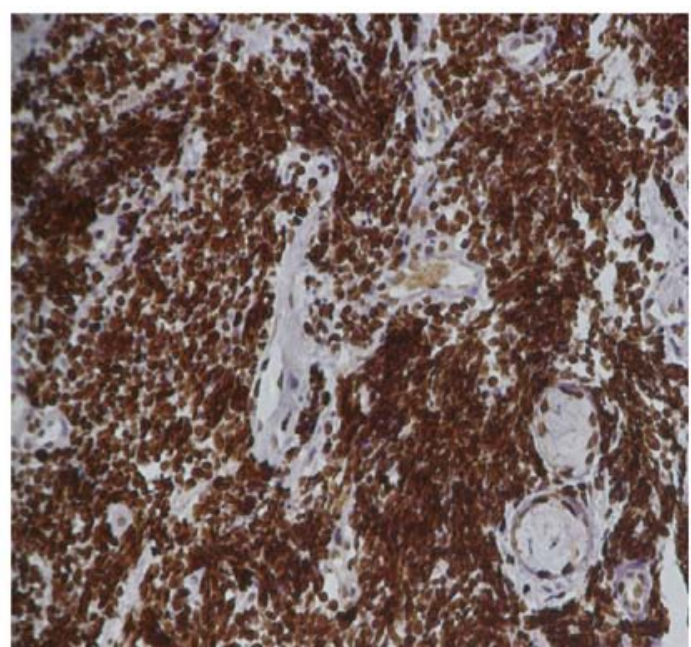

E2F1

Figure 1. Expression levels of $A D A M 12$ and E2F1 in SCLC tissue samples. IHC staining of $A D A M 12$ and E2F1 is shown in normal alveolar epithelial tissue and SCLC tissue samples. PBS instead of the primary antibody was considered as a negative control and VEGFR was considered as a positive control.

weak $(<10 \%)$, moderate $(10-60 \%)$ and strong $(>60 \%)$ positive expression was noted in 2, 3, 12 and 22 cases for $E 2 F 1$; and in 5, 4, 11 and 19 cases for ADAM12 (Table IV). These results were consistent with our previous studies $(8,13)$. The positive expression (including moderate and strong staining) of $E 2 F 1$ and $A D A M 12$ was 85 and $75 \%$, respectively. In the same tissue samples, $A D A M 12$ was highly expressed in the tissue samples for which anti-E2F1 staining was strong-positive (Fig. 1). The section incubated with phosphate-buffered saline (PBS) instead of the primary antibody was considered as the negative control, and the expression of vascular endothelial growth factor receptor (VEGFR) in SCLC has been reported and was considered as the positive control (14), and the expression levels of $E 2 F 1$ and $A D A M 12$ were negative in normal alveolar epithelial cells (Fig. 1).
E2F1 knockdown significantly inhibits the expression of $A D A M 12$. As shown in Fig. 1, E2F1 may regulate the expression of $A D A M 12$. siRNA targeting $E 2 F 1$ was transfected into $\mathrm{H} 1688$ and $\mathrm{H} 446$ cells, and $E 2 F 1$ expression was significantly decreased at the transcription and translation levels (Fig. 2). Meanwhile, $A D A M 12$ expression was also significantly reduced (Fig. 2). This result showed that E2F1 knockdown significantly inhibited the expression of $A D A M 12$ at the mRNA and protein levels, thus indicating that $E 2 F 1$ was able to regulate the expression of $A D A M 12$ at the transcription level.

ChIP-to-seq analysis indicates the binding of E2F1 to $A D A M 12$. As $A D A M 12$ may be regulated by E2F1, ChIPto-seq was employed to discover the $E 2 F 1$ binding sites to 


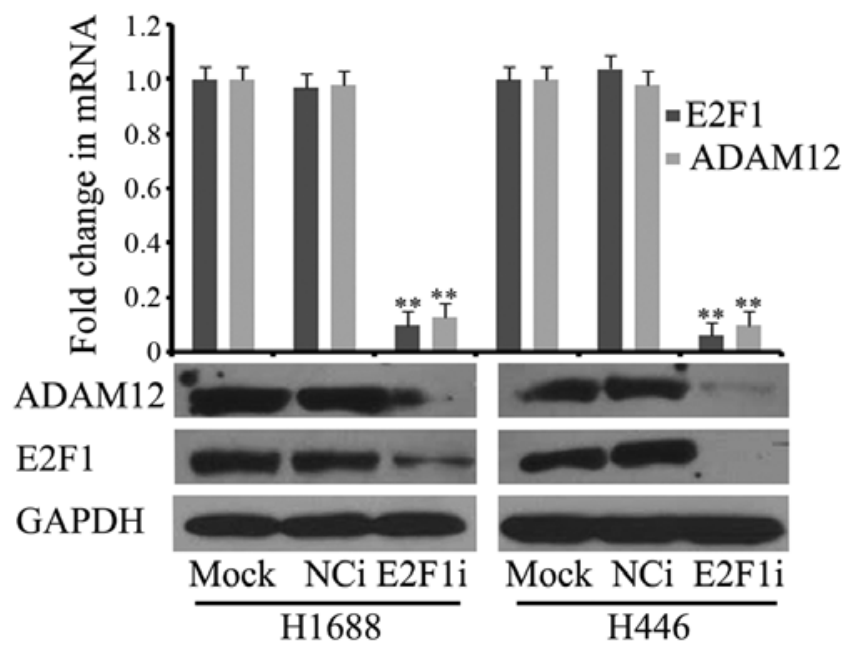

Figure 2. ADAM12 expression is decreased following knockdown of E2F1. The expression of $A D A M 12$ was significantly decreased at the mRNA and protein levels when the expression of $E 2 F 1$ was silenced by siRNA. Mock, untreated cells; NCi, cells treated with the scrambled siRNA; E2F1i, cells treated with the siRNA targeting $E 2 F 1$.

Table V. Features of the $E 2 F 1$ binding DNA fragments in the ADAM12 gene from ChIP-to-seq.

\begin{tabular}{lllc}
\hline Name & $\begin{array}{l}\text { Size } \\
(\mathrm{kb})\end{array}$ & $\mathrm{Ch}$ & Sites \\
\hline ADAM12 & 0.582 & 10 & $128010444-128011026$ \\
& 1.2 & 10 & $128076927-128078127$ \\
& 0.681 & 10 & $128086195-128086876$ \\
\hline
\end{tabular}

Ch, chromosome.

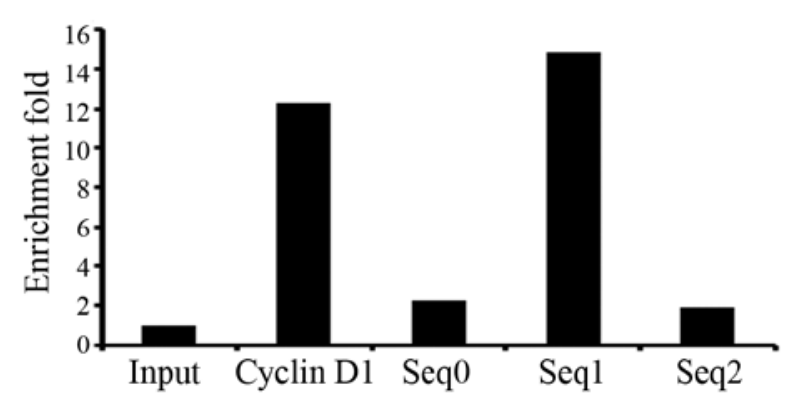

Figure 3. Enrichment fold by $E 2 F 1$ in different fragments. Seq0, seq1 and seq2 could recruit $E 2 F 1$ and the enrichment fold was calculated by qPCR. Cyclin D1 was considered as a positive control.

explore the detailed mechanism. A total of 4,700 genes regulated by $E 2 F 1$ were identified by ChIP-to-seq (data not shown; these data will be reported in a subsequent study). There were three $E 2 F 1$ binding sites in the ADAM12 gene (Table V). They were the following: Chr10: 128010444-128011026, located in the intron of $A D A M 12$, named seq0; Chr10: 128076927128078127, located in the promoter of ADAM12, named seq1; Chr10: 128086195-128086876, located in the upstream $20 \mathrm{~kb}$ from the transcription start site of ADAM12, named seq2.

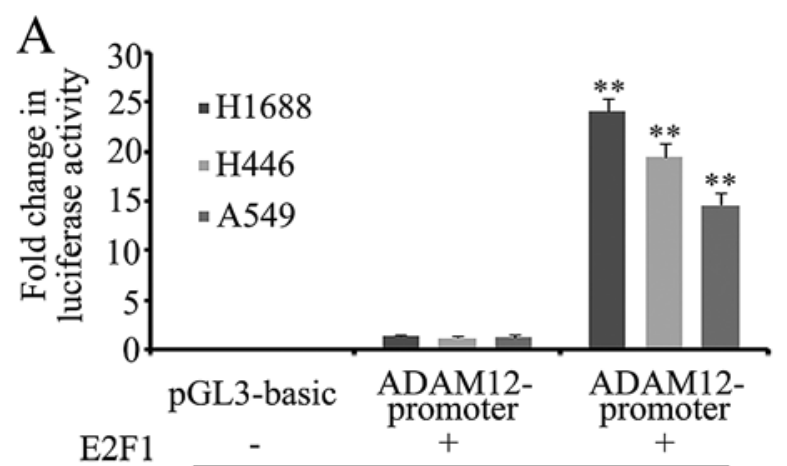

ADAM12-luciferase reporter

B

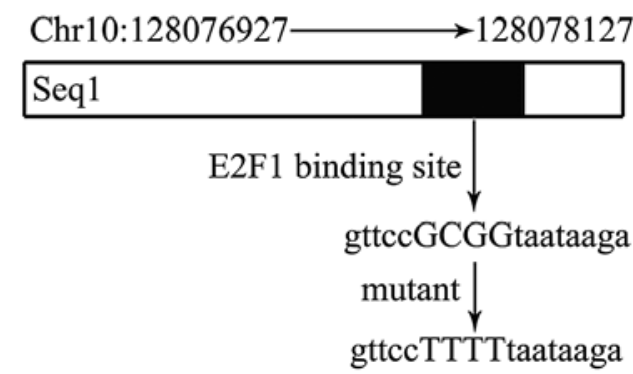

C

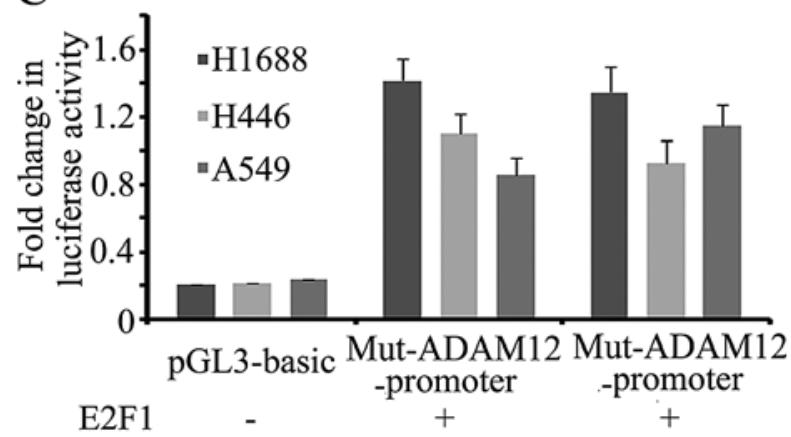

Mut-ADAM12-luciferase reporter

Figure 4. $E 2 F 1$ regulates the expression of $A D A M 12$ by the $E 2 F 1$ binding motif in the promoter region. (A) Transient transfection showed that overexpression of $E 2 F 1$ significantly increased the activity of luciferase driven by the wild-type $A D A M 12$ promoter in $\mathrm{H} 1688, \mathrm{H} 446$ and A549 cells. (B) One putative $E 2 F 1$ binding site was identified by MatInspector software. (C) When $E 2 F 1$ was overexpressed in H1688, H446 and A549 cells, the activity of luciferase driven by the $E 2 F 1$ binding site mutant $A D A M 12$ promoter (mut-ADAM12) was not changed.

Since there were three $E 2 F 1$ binding sites, the enrichment fold was calculated. The results showed that the enrichment fold of seq0, seq1 and seq2 by $E 2 F 1$ was $2.3,14.9$ and 1.9 as determined by qPCR (Fig. 3), indicating that the ability of $E 2 F 1$ binding seq1 was stronger than seq 0 and seq 2 .

E2F1 regulates ADAM12 expression via an E2F1 binding motif in the promoter. After considering the above-mentioned results, we speculated that $E 2 F 1$ could directly regulate $A D A M 12$ expression and tested this using dual-luciferase reporter constructs. The seq1 fragment was cloned and incorporated into the pGL3 basic vector (known as the wild-type $A D A M 12$ promoter). The wild-type $A D A M 12$ promoter was transfected into H1688, H446 and A549 cells, and this significantly promoted the luciferase activity. When co-transfected with the $E 2 F 1$ expression vector, the luciferase activity was 

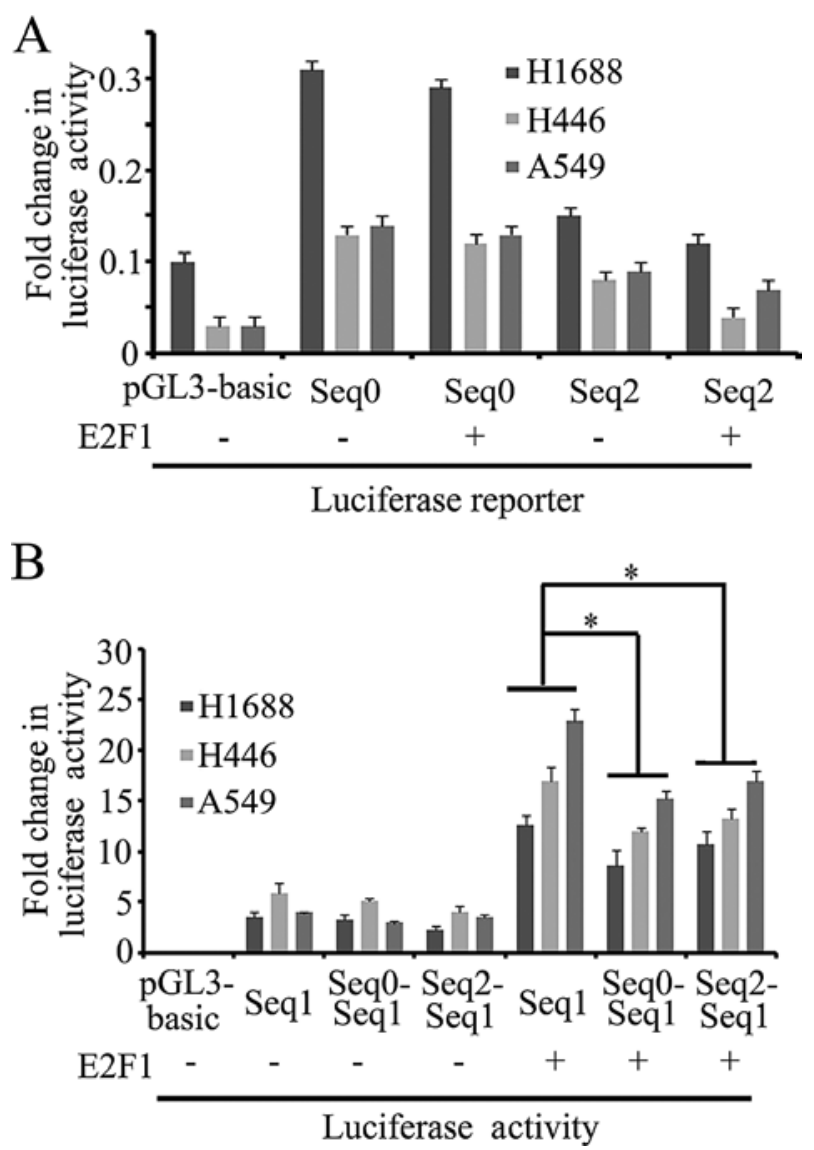

Figure 5. (A) The activity analysis of luciferase driven by the fusion fragments. Seq0 and seq 2 fragments could not drive the activity of luciferase even in the case of the overexpression of E2F1. (B) Compared with seq1, the activities of the fusion fragments were significantly decreased following co-transfection with $E 2 F 1$.

significantly increased (Fig. 4A). One putative $E 2 F 1$ binding site (gttccGCGGtaataaga) was identified by MatInspector software (http://www.genomatix.de/) in the seq1 fragment (Fig. 4B). An $E 2 F 1$ binding site mutant luciferase reporter (known as the mut-ADAM12 promoter) was constructed. Following transfection into H1688, $\mathrm{H} 446$ and A549 cells, overexpression of E2F1 did not significantly promote the activity of the mut- $A D A M 12$ promoter, indicating that $E 2 F 1$ stimulated the expression of $A D A M 12$ via the $E 2 F 1$ binding site in the $A D A M 12$ promoter region (Fig. 4C).

Cis-acting elements seq0 and seq2 inhibit the activity of the ADAM12 promoter. ChIP-to-seq results showed that there were three E2F1 binding sites in the ADAM12 gene (Table V), in which seq1 as a promoter could recruit $E 2 F 1$ to drive $A D A M 12$ expression. Seq0 and seq 2 also bind $E 2 F 1$, but the function of seq0 and seq 2 in the regulation of $A D A M 12$ is unknown. Seq0 and seq2 fragments were cloned into the pGL3 basic vector. After transfection into H1688, H446 and A549 cells, there was no luciferase activity, indicating that seq0 and seq2 fragments could not promote the expression of luciferase (Fig. 5A). We next ascertained whether seq0 or seq2 has enhancer function. Subsequently, the seq0-seq1 and seq2-seq1 fusion luciferase expression constructs were analyzed. Compared with the seq1 fragments, the luciferase activity of fusion fragment vectors was on average decreased, but there was no statistically significant difference. When co-transfected with $E 2 F 1$, the luciferase activity of the fusion fragments was significantly decreased, indicating that seq1 and seq 2 repressed the $A D A M 12$ promoter (Fig. 5B).

\section{Discussion}

ADAM12 exhibits a wide range of expression levels in various tissues and cells (15), but the distribution of $A D A M 12$ is strictly regulated (16). During embryonic development of mice, ADAM12 is expressed in stromal cells and results in bone and muscle development. It is absent in adult rat muscle cells, but is expressed again during the process of muscle regeneration (16-19). In addition, ADAM12 is also expressed in osteoclasts $(20,21)$, macrophages (22), trophoblast cells during embryonic development (23), adipocytes (24), chondrocytes (25) and liver stellate cells (26). These results demonstrate that $A D A M 12$ expression has strict temporal and spatial specificity. Additionally, ADAM12 is highly expressed in certain types of tumors, such as breast (6), prostate (7) and small cell lung cancer (SCLC) (8), but there are limited studies reporting the regulation of $A D A M 12$ expression in tumor tissues. In the present study, $A D A M 12$ and $E 2 F 1$ were highly expressed in the same SCLC tissue samples, indicating that $E 2 F 1$ may control the expression of ADAM12.

$E 2 F 1$, as a classical transcriptional factor, plays important roles in cell cycle regulation, cell proliferation and apoptosis (27). Numerous studies suggest that $E 2 F 1$ is involved in the invasion and metastasis of tumor cells by regulating the expression of matrix metalloproteinases $(13,28)$, thrombospondin1 (29), platelet-derived growth factor receptor (30) and vascular endothelial growth factor receptor (31). The target genes regulated by $E 2 F 1$ in different cells were different when ChIP-on-ChIP or ChIP-to-seq methods were used (32-35). In our ChIP-to-seq database, ADAM12 was able to bind $E 2 F 1$, and $A D A M 12$ expression was most significantly decreased when there was depletion of $E 2 F 1$. Therefore, we explored the detailed mechanism of $A D A M 12$ regulation. Although there are three $E 2 F 1$ binding sites in the $A D A M 12$ gene, their ability for $E 2 F 1$ recruitment differed. One reason may be a difference in the binding motif. The present results showed that $E 2 F 1$ regulated $A D A M 12$ expression via the $E 2 F 1$ binding site in the promoter region, and this was shown to be a functional motif. The other two binding sites were located in the upstream $20-\mathrm{kb}$ and intron regions of $A D A M 12$. They were unable to promote the expression of luciferase, but reduced the activity of the promoter. Although the qPCR results showed that these three elements could recruit $E 2 F 1$, the strongest recruitment was by the promoter sequence. This result possibly indicates that $E 2 F 1$ has a binding site preference due to the different binding motifs.

In conclusion, the present findings offer support that $E 2 F 1$ regulates the expression of $A D A M 12$ by binding the promoter and other cis-acting elements.

\section{Acknowledgements}

The present study was supported by the National Natural Science Foundation of China (grant no. 81302017) and 
the Natural Science Foundation of Shandong (grant no. ZR2013HL004).

\section{References}

1. Mazzocca A, Giannelli G and Antonaci S: Involvement of ADAMs in tumorigenesis and progression of hepatocellular carcinoma: Is it merely fortuitous or a real pathogenic link? Biochim Biophys Acta 1806: 74-81, 2010.

2. Jacobsen J, Visse R, Sørensen HP, Enghild JJ, Brew K, Wewer UM and Nagase H: Catalytic properties of ADAM12 and its domain deletion mutants. Biochemistry 47: 537-547, 2008.

3. Ieguchi K, Tomita T, Omori T, Komatsu A, Deguchi A, Masuda J, Duffy SL, Coulthard MG, Boyd A and Maru Y: ADAM12cleaved ephrin-A1 contributes to lung metastasis. Oncogene 33: 2179-2190, 2014

4. Kerna I, Kisand K, Suutre S, Murde M, Tamm A, Kumm J and Tamm A: The ADAM12 is upregulated in synovitis and postinflammatory fibrosis of the synovial membrane in patients with early radiographic osteoarthritis. Joint Bone Spine 81: 51-56, 2014.

5. Higashiyama S: Membrane-anchored heparin-binding EGF-like growth factor processing by ADAM12 in cardiac hypertrophy. Nihon Rinsho 61: 767-775, 2003 (In Japanese).

6. Roy R, Rodig S, Bielenberg D, Zurakowski D and Moses MA: ADAM12 transmembrane and secreted isoforms promote breast tumor growth: A distinct role for ADAM12-S protein in tumor metastasis. J Biol Chem 286: 20758-20768, 2011.

7. Peduto L, Reuter VE, Sehara-Fujisawa A, Shaffer DR, Scher HI and Blobel CP: ADAM12 is highly expressed in carcinomaassociated stroma and is required for mouse prostate tumor progression. Oncogene 25: 5462-5466, 2006.

8. Shao S, Li Z, Gao W, Yu G, Liu D and Pan F: ADAM-12 as a diagnostic marker for the proliferation, migration and invasion in patients with small cell lung cancer. PLoS One 9: e85936, 2014.

9. Le Pabic H, Bonnier D, Wewer UM, Coutand A, Musso O, Baffet G, Clément B and Théret N: ADAM12 in human liver cancers: TGF-beta-regulated expression in stellate cells is associated with matrix remodeling. Hepatology 37: 1056-1066, 2003

10. Ray BK, Dhar S, Shakya A and Ray A: Z-DNA-forming silencer in the first exon regulates human ADAM-12 gene expression. Proc Natl Acad Sci USA 108: 103-108, 2011.

11. Li H, Solomon E, Duhachek Muggy S, Sun D and Zolkiewska A Metalloprotease-disintegrin ADAM12 expression is regulated by Notch signaling via microRNA-29. J Biol Chem 286 21500-21510, 2011.

12. Díaz B, Yuen A, Iizuka S, Higashiyama S and Courtneidge SA: Notch increases the shedding of HB-EGF by ADAM12 to potentiate invadopodia formation in hypoxia. J Cell Biol 201: 279-292, 2013.

13. Li Z, Guo Y, Jiang H, Zhang T, Jin C, Young CY and Yuan H: Differential regulation of MMPs by E2F1, Sp1 and NF-kappa B controls the small cell lung cancer invasive phenotype. BMC Cancer 14: 276, 2014.

14. Ioannou M, Papamichali R, Kouvaras E, Mylonis I, Vageli D, Kerenidou T, Barbanis S, Daponte A, Simos G, Gourgoulianis K, et al: Hypoxia inducible factor-1 alpha and vascular endothelial growth factor in biopsies of small cell lung carcinoma. Lung 187: 321-329, 2009.

15. Harris HA, Murrills RJ and Komm BS: Expression of meltrinalpha mRNA is not restricted to fusagenic cells. J Cell Biochem 67: 136-142, 1997.

16. Kurisaki T, Masuda A, Osumi N, Nabeshima Y and FujisawaSehara A: Spatially- and temporally-restricted expression of meltrin alpha (ADAM12) and beta (ADAM19) in mouse embryo. Mech Dev 73: 211-215, 1998.
17. Borneman A, Kuschel R and Fujisawa-Sehara A: Analysis for transcript expression of meltrin alpha in normal, regenerating, and denervated rat muscle. J Muscle Res Cell Motil 21: 475-480, 2000.

18. Galliano MF, Huet C, Frygelius J, Polgren A, Wewer UM and Engvall E: Binding of ADAM12, a marker of skeletal muscle regeneration, to the muscle-specific actin-binding protein, alpha -actinin-2, is required for myoblast fusion. J Biol Chem 275: 13933-13939, 2000.

19. Cao Y, Zhao Z, Gruszczynska-Biegala J and Zolkiewska A: Role of metalloprotease disintegrin ADAM12 in determination of quiescent reserve cells during myogenic differentiation in vitro. Mol Cell Biol 23: 6725-6738, 2003.

20. Abe E, Mocharla H, Yamate T, Taguchi Y and Manolagas SC: Meltrin-alpha, a fusion protein involved in multinucleated giant cell and osteoclast formation. Calcif Tissue Int 64: 508-515, 1999.

21. Verrier S, Hogan A, McKie N and Horton M: ADAM gene expression and regulation during human osteoclast formation. Bone 35: 34-46, 2004

22. Wu C, Li L, Zhao J, Fan Q, Tian WX and He RQ: Effect of $\alpha_{2} \mathrm{M}$ on earthworm fibrinolytic enzyme III-1 from Lumbricus rubellus. Int J Biol Macromol 31: 71-77, 2002.

23. Gilpin BJ, Loechel F, Mattei MG, Engvall E, Albrechtsen R and Wewer UM: A novel, secreted form of human ADAM 12 (meltrin alpha) provokes myogenesis in vivo. J Biol Chem 273: 157-166, 1998.

24. Tani N, Higashiyama S, Kawaguchi N, Madarame J, Ota I, Ito Y, Ohoka Y, Shiosaka S, Takada Y and Matsuura N: Expression level of integrin alpha 5 on tumour cells affects the rate of metastasis to the kidney. Br J Cancer 88: 327-333, 2003.

25. Kveiborg M, Albrechtsen R, Rudkjaer L, Wen G, DamgaardPedersen K and Wewer UM: ADAM12-S stimulates bone growth in transgenic mice by modulating chondrocyte proliferation and maturation. J Bone Miner Res 21: 1288-1296, 2006.

26. Bourd-Boittin K, Le Pabic H, Bonnier D, L'Helgoualc'h A and Théret N: RACK1, a new ADAM12 interacting protein. Contribution to liver fibrogenesis. J Biol Chem 283: 26000-26009, 2008.

27. Wyllie AH: E2F1 selects tumour cells for both life and death. J Pathol 198: 139-141, 2002.

28. Johnson JL, Pillai S, Pernazza D, Sebti SM, Lawrence NJ and Chellappan SP: Regulation of matrix metalloproteinase genes by E2F transcription factors: Rb-Raf-1 interaction as a novel target for metastatic disease. Cancer Res 72: 516-526, 2012.

29. Ji W, Zhang W and Xiao W: E2F-1 directly regulates thrombospondin 1 expression. PLoS One 5: e13442, 2010.

30. Minato Y, Tashiro E, Kanai M, Nihei Y, Kodama Y and Imoto M: Transcriptional regulation of a new variant of human platelet-derived growth factor receptor alpha transcript by E2F-1. Gene 403: 89-97, 2007.

31. Pillai S, Kovacs M and Chellappan S: Regulation of vascular endothelial growth factor receptors by $\mathrm{Rb}$ and $\mathrm{E} 2 \mathrm{~F} 1$ : Role of acetylation. Cancer Res 70: 4931-4940, 2010.

32. Lavrrar JL and Farnham PJ: The use of transient chromatin immunoprecipitation assays to test models for E2F1-specific transcriptional activation. J Biol Chem 279: 46343-46349, 2004.

33. Jin VX, Rabinovich A, Squazzo SL, Green R and Farnham PJ: A computational genomics approach to identify cis-regulatory modules from chromatin immunoprecipitation microarray data - a case study using E2F1. Genome Res 16: 1585-1595, 2006.

34. Weinmann AS, Bartley SM, Zhang T,Zhang MQ and Farnham PJ: Use of chromatin immunoprecipitation to clone novel E2F target promoters. Mol Cell Biol 21: 6820-6832, 2001.

35. Wells J, Graveel CR, Bartley SM, Madore SJ and Farnham PJ: The identification of E2F1-specific target genes. Proc Natl Acad Sci USA 99: 3890-3895, 2002. 